\title{
BER Evaluation of OFDM Systems with Joint Effect of TI-ADC Circuit's Gain Mismatch and Channel Estimation Error
}

\author{
Vo-Trung-Dung Huynh, Nele Noels, Member, IEEE, and Heidi Steendam, Senior Member, IEEE
}

\begin{abstract}
The identification of maximum tolerable levels for potential mismatches is critical when designing communication systems. In this paper, we derive maximum tolerable levels for time-interleaved analog-to-digital-converter (TI-ADC) gain mismatch in orthogonal frequency division multiplexing (OFDM) systems. To this end, we first analytically evaluate the bit error rate (BER) for square quadrature amplitude modulatedOFDM (QAM-OFDM) systems that are impaired by (i) the gain mismatch of a TI-ADC and (ii) the channel estimation errors (CEEs) of a zero-forcing equalizer. Our analysis includes the cases of a frequency-selective Rayleigh fading channel and a wired channel. Next, built on the obtained BER expressions, a threshold is established on the gain mismatch level, at which an error floor caused by the gain mismatch is below a given BER value at high signal-to-noise ratios (SNRs) in the absence of CEEs. Finally, numerical results further show that if and only if we set the gain mismatch level below 0.25 of this threshold, there is essentially no BER performance degradation compared with the mismatch-free case.
\end{abstract}

Index Terms-Bit error rate, OFDM, TI-ADC, gain mismatch, channel estimation error, square QAM, Rayleigh channels, wired channels.

\section{INTRODUCTION}

Orthogonal frequency division multiplexing (OFDM) is an efficient data modulation technique that is extensively used for many broadband wired and wireless communication systems to mitigate the effects of delay spread in dispersive channels [1]. For instance, current multi-Gigabit fiber-optic communication systems employ OFDM to increase the data transmission rates to $100 \mathrm{Gbps}$ and beyond [2]. OFDM has also received growing attention in emerging ultra-high speed wireless communication systems including ultra-wideband systems in the 3.1-10.6 GHz band [3] and millimeter-wave systems in the $60 \mathrm{GHz}$ band (e.g., unlicensed spectrum from $57-64 \mathrm{GHz}$ available in the US) [4]. Such high-speed OFDM systems require that the receiver is equipped with a high sampling rate analog-to-digital converter (ADC), which is placed prior to the baseband digital signal processing unit. Since the operating sampling rate of a regular ADC is limited by the physical constrains of the current technology [5], a time-interleaved (TI) architecture is frequently employed.

To obtain a sampling rate $\frac{1}{T_{s}}$, a TI-ADC is constructed with $L$ identical sub-ADCs, each sampling the analog input signal at a lower rate $\frac{1}{L T_{s}}$, i.e., the $l$-th sub-ADC samples the input signal at time instants $t_{k}^{(l)}=l T_{s}+k L T_{s}$, where $l=0,1, \ldots, L-1, k=0,1,2, \ldots$. Unfortunately, due to component inequalities and tiny asymmetries in ADC chip layouts causing unknown offset, gain and timing mismatches between the sub-ADC outputs, the use of a TI-ADC can significantly degrade the overall system performance. The effect of these mismatches and mismatch calibration approaches have been intensively studied for single-carrier systems over the past decades [6]-[13]. More recently, these issues have also been investigated for high-speed OFDM systems [14][17]. In general, it was shown that TI-ADC mismatches affect OFDM systems in quite a different way than single-carrier systems. Most of these works, however, have investigated the effect of TI-ADC mismatches on OFDM bit error rate (BER) through numerical simulation only. This motivated further research on approaches to derive simple TI-ADC mismatchimpaired BER expressions which can be evaluated efficiently and which can be investigated by analytical means in order to understand some of the main processes at work. As a first step, a full study on the effect of offset mismatch was reported in [18]. As a follow-up, the present paper is concerned with the impact of gain mismatch. As opposed to offset mismatch, gain mismatch causes inter-carrier interference (ICI) [15], [17], [19] which can severely degrade the performance of an OFDM transmission. Hence, the impact of gain mismatch is potentially larger than that of offset mismatch. Parts of the work on gain mismatch have been published previously [19], [20]. In [19], we used a Gaussian approach (GA) to the ICI caused by gain mismatch. The study considered an additive white Gaussian noise (AWGN) channel only, in which case the GA was seen to produce an inaccurate estimate of the true BER for small values of $L$. As an alternative, in [20], we proposed to use a semi-analytic approach (SA). Simulation results in [20] have confirmed the accuracy of the SA for any value of $L$ and for AWGN as well as for frequency-selective fading channels. Major drawbacks of the SA as compared to the GA are (i) the complexity of the associated BER evaluation procedure and (ii) the lack of insight provided by the obtained BER expressions. We note that, until now, the accuracy of the GA has never been investigated for frequency-selective fading channels, while OFDM systems are often specifically designed for such channels [1]. In OFDM systems, a frequency-selective channel is converted into a collection of flat fading channels, and therefore its effect can be compensated by simply using a one-tap frequency-domain equalizer per sub-carrier [2]. As in many coherent communication systems, channel estimation errors (CEEs) have a direct impact on the bit error rate (BER) performance of the OFDM system. The isolated influence of such CEEs on the BER performance for some modulation 
TABLE I

NOTATIONS AND CONVENTIONS

\begin{tabular}{|c|l|}
\hline Notation & Meaning \\
\hline$x^{*}$ & complex conjugate of $x$ \\
\hline $\mathbf{X}^{T}$ & transpose of vector $\mathbf{X}$ \\
\hline$N$ & FFT size \\
\hline$N_{C P}$ & cyclic prefix length \\
\hline$L$ & number of sub-ADCs \\
\hline$E_{s}$ & symbol energy \\
\hline$I_{N}$ & $\{0,1, \ldots, N-1\}$ \\
\hline$I_{L}$ & $\{0,1, \ldots, L-1\}$ \\
\hline$N_{0}$ & noise power spectral density \\
\hline$d g_{l}$ & gain error for the $l^{\text {th }}$ sub-ADC \\
\hline$d g_{l}^{x \%}$ & $\begin{array}{l}\text { gain error for the } l^{\text {th }} \text { sub-ADC if mismatch level is } \\
\text { at } x\end{array}$ \\
\hline$X \sim\left(\mu, 2 \sigma^{2}\right)$ & $\begin{array}{l}X \text { is complex-valued circularly symmetric Gaussian } \\
\text { distributed with mean } \mu \text { and variance } \sigma^{2} \text { per } \\
\text { dimension }\end{array}$ \\
\hline$p_{x}(x)$ & probability density function (pdf) of $x$ \\
\hline$\Re\{x\}$ & real part of $x$ \\
\hline$\Im\{x\}$ & imaginary part of $x$ \\
\hline$\delta_{k}$ & Kronecker delta function \\
\hline $\bmod (x, y)$ & $\begin{array}{l}\text { value in }[0, y) \text { such that } x=i y+\bmod (x, y) \text { with } \\
i \text { integer }\end{array}$ \\
\hline$|x|$ & absolute value of $x$ \\
\hline
\end{tabular}

orders and types, i.e., if no other disturbances are present except the CEE, was studied in [21]-[23] for single-carrier systems, and in [24]-[26] for multi-carrier systems. In particular, in [26], an approximate closed-form BER expression in the presence of CEEs was derived for square QAM-OFDM systems, but this approximation is valid for a small range of the CEE variance only.

In this paper, we consider the joint effect of TI-ADC gain mismatch and CEEs on the OFDM BER performance in frequency-selective Rayleigh fading and wired channels. To this end, we use the GA. We show that the obtained BER expressions can be evaluated efficiently, while providing a good approximation of the true BER. Regarding the impact of the CEEs, the derived BER expression is more accurate than the one proposed in [26]. Further, as far as TI-ADC gain mismatch is concerned, the analytical BER expression for wired channels is less accurate than for Rayleigh channels. The difference between the Rayleigh channels and the wired channels is thoroughly discussed. As top of the bill, we derive a rule-of-thumb for determining the maximum tolerable gain mismatch level (in the absence of CEEs). This is the largest gain mismatch level for which the BER performance degradation with respect to the mismatch-free case remains below an acceptable limit. Maximum tolerable gain mismatch levels serve as important guidelines for circuit-and-system design engineers to compensate the gain mismatch through hardware calibration or digital signal processing [15], [27], [28].

The paper is organized as follows. First, Table I lists the notations used throughout the paper. Then, Section II describes the system model. The BER expressions for Rayleigh and wired channels are derived in Section III. Square QAM and binary reflected Gray code (BRGC) bit mapping [29] are assumed. The results for pulse amplitude modulation (PAM) follow as a special case. In Section IV, we validate the accuracy of the obtained expressions by comparing their numerical

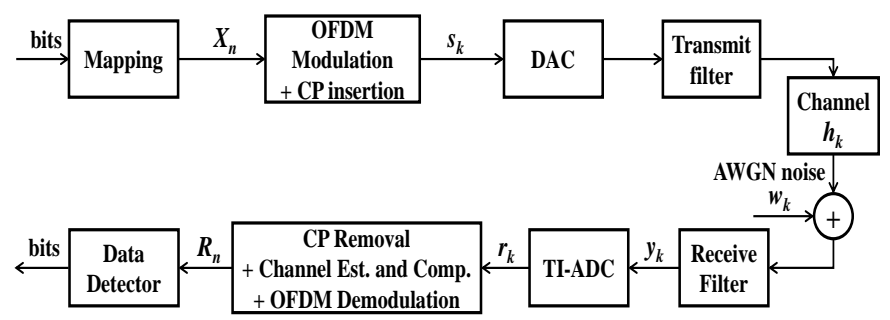

Fig. 1. Block diagram of an OFDM system with a TI-ADC at the receiver.

evaluation with the results of a brute-force Monte Carlo (MC) simulation. We derive a rule-of-thumb for a tolerable gain mismatch level inducing a negligible BER performance degradation in the case of fixed gain mismatch in Section $\mathrm{V}$, and in the case of random gain mismatch in Section VI. Finally, Section VII presents the conclusions of the study.

\section{SySTEM MODEL}

\section{A. Transmitter}

The OFDM system under consideration is shown in Fig. 1. The receiver is assumed to employ a TI-ADC. The different sub-ADCs of the TI-ADC experience different gain errors. To simplify the notations, we consider the transmission of a single OFDM block $\mathbf{X}$ consisting of $N$ complex-valued data symbols in the frequency domain, i.e., $\mathbf{X}=\left(X_{0}, X_{1}, \ldots, X_{N-1}\right)^{T}$ that are taken from a unit-energy square $M^{2}$-QAM constellation $\Omega$. Each complex-valued constellation symbol is equivalent to the orthogonal superposition of 2 real-valued PAM symbols (I and $\mathrm{Q}$ components), each corresponding to a sequence of $m=\log _{2} M$ input data bits according to the BRGC mapping rule [29]. The vector $\mathbf{X}$ is applied to an inverse discrete Fourier transform (IDFT) of size $N$. The resulting time-domain samples are extended with a cyclic prefix (CP) of length $N_{C P}$, which protects the received OFDM symbol against inter-symbol interference (ISI) caused by the frequency selectivity of the channel. The time-domain samples $s_{k}$ are given by

$$
s_{k}=\frac{1}{\sqrt{N}} \sum_{n \in I_{N}} X_{n} e^{j 2 \pi \frac{n k}{N}},-N_{C P} \leq k \leq N-1 .
$$

Before transmitting over the channel $h_{k}$ and adding an AWGN noise $w_{k}$, these samples are passed through a digital-to-analog converter (DAC) and a transmit filter.

\section{B. Receiver}

At the receiver, we assume perfect timing synchronization and matched filtering. After passing through the receive filter, the received waveform is sampled at the Nyquist rate $\frac{1}{T_{s}}$ by a TI-ADC with $L$ parallel sub-ADCs. The TI-ADC is assumed to have a sufficiently high resolution, so the quantization noise can be neglected [8], [15], [30]. Further, since in practice, the gain errors of the sub-ADCs in a TI-ADC vary only very slowly with time [15], we model them as constants over an OFDM symbol period. Using the model from [15], the output of the TI-ADC with gain mismatch can be written as

$$
r_{k}=\left(1+d g_{l}\right) y_{k}, \quad-N_{C P} \leq k \leq N-1,
$$


where $l=\bmod (k, L), d g_{l}$ is the gain error of the $l$-th subADC, expressed relative to the transmitted symbol energy $E_{s}$, and $y_{k}$ is given by

$$
\begin{aligned}
y_{k} & =\sqrt{E_{s}} \sum_{m=0}^{N_{C P}-1} h_{m} s_{k-m}+w_{k} \\
& =\frac{\sqrt{E_{s}}}{\sqrt{N}} \sum_{n \in I_{N}} X_{n} \sum_{m=0}^{N_{C P}-1} h_{m} e^{-j 2 \pi \frac{m n}{N}} e^{j 2 \pi \frac{n k}{N}} \\
& +\frac{1}{\sqrt{N}} \sum_{n \in I_{N}} W_{n} e^{j 2 \pi \frac{n k}{N}} \\
& =\frac{1}{\sqrt{N}} \sum_{n \in I_{N}}\left(\sqrt{E_{s}} X_{n} H_{n}+W_{n}\right) e^{j 2 \pi \frac{n k}{N}} .
\end{aligned}
$$

Here, $w_{k}=\frac{1}{\sqrt{N}} \sum_{n \in I_{N}} W_{n} e^{j 2 \pi \frac{n k}{N}}$, with $W_{n} \sim \aleph\left(0, N_{0}\right)$ independent and identically distributed (i.i.d.) Gaussian noise samples, and $H_{n}=\sum_{m=0}^{N_{C P}-1} h_{m} e^{-j 2 \pi \frac{m n}{N}}$ are the channel coefficients in the frequency domain. In this paper, we consider the cases of a slowly-varying multi-path Rayleigh fading channel and a wired channel. In both cases, the channel remains roughly constant over an OFDM symbol duration.

The receiver removes the $\mathrm{CP}$ and converts the remaining $N$ samples to the frequency domain using a discrete Fourier transform (DFT). Before data detection, the receiver employs a zero-forcing $(\mathrm{ZF})$ equalizer to compensate for the channel [25]. The output of the equalizer at the $n$-th sub-carrier is given by

$$
R_{n}=\frac{1}{\hat{H}_{n} \sqrt{N}} \sum_{k \in I_{N}} r_{k} e^{-j 2 \pi \frac{k n}{N}},
$$

where $\hat{H}_{n}$ denotes the estimate of $H_{n}$. The quantities $R_{n}$ (4) are used to perform bit sequence detection by mapping them to the nearest constellation point and applying the inverse mapping rule.

\section{APPROXIMATE BER EXPRESSION USING GA}

In this section, we derive a simple approximate BER expression for the considered system. In the derivation, we need appropriate statistical models for the ICI caused by gain mismatch and for the CEE caused by channel estimation, and this for given data symbols $X_{n}$ and channel estimates $\hat{H}_{n}$.

\section{A. Channel estimation error}

Let us consider a fixed gain mismatch, and a given but further unspecified estimator. We employ a general additive estimation error model for the channel estimate $\hat{H}_{n}$ in (4) [25], i.e.,

$$
\hat{H}_{n}=H_{n}+U_{n},
$$

where the independent $\mathrm{CEE} U_{n} \sim \aleph\left(0,2 \sigma_{U}^{2}\right)$, and $U_{n}$ is statistically independent of $H_{n}$ and $\hat{H}_{n}$ [25], [26]. The value of $\sigma_{U}^{2}$ reflects the level of the channel estimation accuracy. Depending on the type of channel, we distinguish two cases:

1) In the case of a wireless RF communication system, where the channel can be described by a Rayleigh fading channel, with $H_{n} \sim \aleph\left(0,2 \sigma_{H}^{2}\right)$, the channel estimates $\hat{H}_{n}$ (5) can be modelled as circularly symmetric complex Gaussian random variables with zero mean and variance $\sigma_{\hat{H}}^{2}$ per dimension, with $\sigma_{\hat{H}}^{2}=\sigma_{H}^{2}+\sigma_{U}^{2}$. As a result,
$\left|\hat{H}_{n}\right|$ has a Rayleigh distribution with probability density function (pdf)

$$
p_{\left|\hat{H}_{n}\right|}\left(\left|\hat{H}_{n}\right|\right)=\frac{\left|\hat{H}_{n}\right|}{\sigma_{\hat{H}}^{2}} e^{-\frac{\left|\hat{H}_{n}\right|^{2}}{2 \sigma_{\hat{H}}^{2}}} .
$$

2) In the case of a fibre-optic or wired RF communication system, the channel can be modelled as a static channel over multiple OFDM symbols, i.e., $H_{n}$ are constants. In that case, the channel estimates $\hat{H}_{n}(5)$ can be modelled as independent circularly symmetric complex Gaussian random variables with mean $H_{n}$ and variance $\sigma_{U}^{2}$ per dimension. Consequently, $\left|\hat{H}_{n}\right|$ has a Rice distribution with pdf

$$
p_{\left|\hat{H}_{n}\right|}\left(\left|\hat{H}_{n}\right|\right)=\frac{\left|\hat{H}_{n}\right|}{\sigma_{U}^{2}} e^{-\frac{\left|\hat{H}_{n}\right|^{2}+\left|H_{n}\right|^{2}}{2 \sigma_{U}^{2}}} J_{0}\left(\frac{\left|H_{n}\right|}{\sigma_{U}^{2}}\left|\hat{H}_{n}\right|\right) \text {, }
$$

where $J_{0}(z)$ is the modified zero-th order Bessel function of the first kind.

\section{B. Interference-plus-noise and CEE terms}

In this subsection, we derive a simple statistical model for the ICI term that results from substituting (2) and (3) into (4). In order to derive a closed-form expression for this term, we first introduce the window function $\pi_{k}$ that equals 1 for $k \in I_{N}$ and 0 otherwise, and whose discrete-time Fourier transform (DTFT) $\Pi(F)$ is given by

$$
\begin{aligned}
\Pi(F) & =N \sum_{k=-\infty}^{\infty} \operatorname{sinc}(N(F-k)) e^{-j \pi N(F-k)} \\
& \approx N \operatorname{sinc}\left([N F]_{-\frac{N}{2}, \frac{N}{2}}\right) e^{-j \pi[N F]_{-\frac{N}{2}}, \frac{N}{2}}
\end{aligned}
$$

for large $N$, where $[x]_{-\frac{N}{2}, \frac{N}{2}}$ denotes $\bmod \left(x+\frac{N}{2}, N\right)-\frac{N}{2}$. Substituting (2) and (3) into (4), using $\pi_{k}$ to extend the summation over $k$ to $k \in[-\infty, \infty]$, and replacing this summation by a summation over $q L+l$ with $q \in[-\infty,+\infty]$ and $l \in I_{L}$, we obtain

$$
\begin{aligned}
R_{n}= & \frac{1}{\hat{H}_{n} N} \sum_{a \in I_{N}}\left(\sqrt{E_{s}} X_{a} H_{a}+W_{a}\right) \\
& \times \sum_{l \in I_{L}}\left(1+d g_{l}\right) e^{-j 2 \pi \frac{(n-a) l}{N}} \sum_{q=-\infty}^{+\infty} \pi_{q L+l} e^{-j 2 \pi \frac{(n-a) L q}{N}} .
\end{aligned}
$$

Taking into account that the last summation in (9) is the DTFT of a sub-sampled and time-shifted version of $\pi_{k}$ evaluated in $F=\frac{(n-a) L}{N}$, we obtain

$$
\begin{aligned}
e^{-j 2 \pi \frac{(n-a) l}{N}} \sum_{q=-\infty}^{+\infty} \pi_{q L+l} e^{-j 2 \pi \frac{(n-a) L q}{N}} & \\
& =\frac{1}{L} \sum_{i \in I_{L}} \Pi\left(\frac{(n-a)}{N}-\frac{i}{L}\right) e^{-j 2 \pi \frac{i l}{L}} .
\end{aligned}
$$

Substituting (8) and (10) into (9), we obtain after appropriate rearrangements

$$
\begin{aligned}
& R_{n} \approx \frac{1}{\hat{H}_{n}}\left(1+D G_{0}\right)\left(\sqrt{E_{s}} X_{n} H_{n}+W_{n}\right) \\
& +\frac{1}{\hat{H}_{n}} \sum_{i \in I_{L} \backslash 0} D G_{i} \sum_{a \in I_{N}, a \neq n}\left(\sqrt{E_{s}} X_{a} H_{a}+W_{a}\right) f\left(a-p_{i, n}\right),
\end{aligned}
$$


where the approximation holds for large $N$ and large $\frac{N}{L}$. In (11), $D G_{i}, f(z)$ and $p_{i}$ are defined as

$$
\begin{gathered}
D G_{i}=\frac{1}{L} \sum_{l \in I_{L}} d g_{l} e^{-j 2 \pi \frac{i l}{L}}, \\
f(z)=\frac{\sin (\pi z)}{\pi z} e^{-j \pi z}
\end{gathered}
$$

and

$$
p_{i, n}=\bmod \left(n-i \frac{N}{L}, N\right),
$$

respectively. Further, in arriving at (11), we have neglected the term $\sum_{i \in I_{L} \backslash 0} D G_{i} f\left(n-p_{i, n}\right)$ which is very small in the usual case of large $\frac{N}{L}$.

Substituting (5) into (11), $R_{n}$ in (11) can be further simplified as

$$
R_{n} \approx \sqrt{E_{s}}\left(1+D G_{0}\right) X_{n}+\Lambda_{n} .
$$

In (15), the interference-plus-noise term $\Lambda_{n}$ is given by

$$
\Lambda_{n}=\Lambda_{1, n}+\Lambda_{2, n}+\Lambda_{3, n},
$$

where the interference term $\Lambda_{1, n}$ and the noise term $\Lambda_{2, n}$ caused by the gain mismatch only, and the interference term $\Lambda_{3, n}$ caused by both CEE and gain mismatch, are defined as

$$
\begin{aligned}
\Lambda_{1, n}= & \frac{\sqrt{E_{s}}}{\hat{H}_{n}} \sum_{i \in I_{L} \backslash 0} D G_{i} \sum_{a \in I_{N}, a \neq n} X_{a} H_{a} f\left(a-p_{i, n}\right), \\
\Lambda_{2, n} & =\frac{1}{\hat{H}_{n}}\left(1+D G_{0}\right) W_{n} \\
& +\frac{1}{\hat{H}_{n}} \sum_{i \in I_{L} \backslash 0} D G_{i} \sum_{a \in I_{N}, a \neq n} W_{a} f\left(a-p_{i, n}\right)
\end{aligned}
$$

and

$$
\Lambda_{3, n}=-\frac{\sqrt{E_{s}}}{\hat{H}_{n}}\left(1+D G_{0}\right) X_{n} U_{n},
$$

respectively. Note that for integer ratios $\frac{N}{L}, p_{i, n}$ is integer valued. As a result, $f\left(a-p_{i, n}\right)=\delta_{a-p_{i, n}}$. Hence, (17) and (18) reduce to $\Lambda_{1, n}=\frac{\sqrt{E_{s}}}{\hat{H}_{n}} \sum_{i \in I_{L} \backslash 0} D G_{i} X_{p_{i, n}} H_{p_{i, n}}$ and $\Lambda_{2, n}=$ $\frac{1}{\hat{H}_{n}}\left(1+D G_{0}\right) W_{n}+\frac{1}{\hat{H}_{n}} \sum_{i \in I_{L} \backslash 0} D G_{i} W_{p_{i, n}}$.

Modelling $X_{n}$ as i.i.d. random variables with zero mean and unit variance, and as $\hat{H}_{n}$ is assumed to be independent of $H_{n^{\prime}}$ for $n \neq n^{\prime 1}$, it immediately follows that (for a given $X_{n}$ and $\left.\hat{H}_{n}\right) \Lambda_{n}$ is (approximately) circularly symmetric complex Gaussian distributed with zero mean and variance $\sigma_{\Lambda}^{2}$ per dimension. Indeed:

1) $\Lambda_{1, n}, \Lambda_{2, n}$ and $\Lambda_{3, n}$ are statistically independent with zero mean.

2) $\Lambda_{1, n}$ is (approximately) circularly symmetric complex Gaussian distributed.

- In the case of Rayleigh fading channels (i.e., $H_{n} \sim$ $\left.\aleph\left(0,2 \sigma_{H}^{2}\right)\right)$, the terms in (17) are Gaussian distributed (as the product of a Gaussian and a discrete random variable). As a result, $\Lambda_{1, n}$ itself has a Gaussian distribution.

${ }^{1}$ Hence, we have $E\left\{\frac{H_{n^{\prime}}}{\hat{H}_{n}} \mid \hat{H}_{n}\right\}=\frac{1}{\hat{H}_{n}} E\left\{H_{n^{\prime}}\right\}$ for $n \neq n^{\prime}$.
- In the case of static channels (i.e., $H_{n}$ are constants), (17) is a linear combination of i.i.d. discrete random variables. Taking into account the generalized central limit theorem [31], $\Lambda_{1, n}$ can nevertheless be approximated as a complex Gaussian distributed random variable, if for non-integer ratios $\frac{N}{L}$, either $L$ or $N$ is sufficiently large; and for integer ratios $\frac{N}{L}, L$ is sufficiently large. In Section IV, we study what happens if these conditions are not fulfilled.

The variance of $\Lambda_{1, n}$ is given by

$$
\begin{aligned}
\sigma_{\Lambda_{1, n}}^{2}= & \frac{E_{s}}{\left|\hat{H}_{n}\right|^{2}} \sum_{i_{1}, i_{2} \in I_{L} \backslash 0} D G_{i_{1}}\left(D G_{i_{2}}\right)^{*} \\
& \times \sum_{a \in I_{N}, a \neq n} A_{a} f\left(a-p_{i_{1}, n}\right) f\left(a-p_{i_{2}, n}\right)
\end{aligned}
$$

per dimension, where

$$
A_{a}=\left\{\begin{array}{cl}
\sigma_{H}^{2} & , \text { Rayleigh channel } \\
\frac{1}{2}\left|H_{a}\right|^{2} & , \text { wired channel. }
\end{array}\right.
$$

3) $\Lambda_{2, n}$ and $\Lambda_{3, n}$ are circularly symmetric complex Gaussian distributed random variables with respective variances per dimension

$$
\begin{aligned}
\sigma_{\Lambda_{2, n}}^{2}= & \frac{N_{0}}{2\left|\hat{H}_{n}\right|^{2}}\left(1+D G_{0}\right)^{2} \\
& +\frac{N_{0}}{2\left|\hat{H}_{n}\right|^{2}} \sum_{i_{1}, i_{2} \in I_{L} \backslash 0} D G_{i_{1}}\left(D G_{i_{2}}\right)^{*} \\
& \quad \times \sum_{a \in I_{N}, a \neq n} f\left(a-p_{i_{1}, n}\right) f\left(a-p_{i_{2}, n}\right)
\end{aligned}
$$

and

$$
\sigma_{\Lambda_{3, n}}^{2}=\frac{E_{s}}{\left|\hat{H}_{n}\right|^{2}}\left(1+D G_{0}\right)^{2}\left|X_{n}\right|^{2} \sigma_{U}^{2} .
$$

It follows that

$$
\sigma_{\Lambda}^{2}\left(X_{n}, \hat{H}_{n}\right)=\sigma_{\Lambda_{1, n}}^{2}+\sigma_{\Lambda_{2, n}}^{2}+\sigma_{\Lambda_{3, n}}^{2}
$$

per dimension. For Rayleigh channels, this model is exact. For wired channels, this model is only an approximation.

\section{BER expression}

In this subsection, we derive the BER of an OFDM system in a Rayleigh or wired channel for fixed gain errors and a fixed CEE variance. If $\Lambda_{n} \sim \aleph\left(0,2 \sigma_{\Lambda}^{2}\left(X_{n}, \hat{H}_{n}\right)\right)$, then for given $X_{n}$, given $\left\{D G_{i}\right\}_{i \in I_{L}}$ and given $\hat{H}_{n}$, the conditional BER for a given channel estimate vector $\hat{\mathbf{H}}$ can be obtained straightforwardly according to the well-established error-rate results for an AWGN channel [19], [32]. Assuming $N_{d}$ datamodulated sub-carriers ${ }^{2}$, we obtain

$$
B E R_{\mid \hat{\mathbf{H}}}=\frac{1}{N_{d} m M^{2}} \sum_{n, u, X_{n}, y} \lambda_{u, \Re\left\{X_{n}\right\}, y} \operatorname{erfc}\left(\Upsilon_{u, X_{n}, y}\left(\left|\hat{H}_{n}\right|\right)\right),
$$

\footnotetext{
${ }^{2}$ In practice, in many OFDM systems, not all $N$ sub-carriers are used for data transmission. For instance, a few sub-carriers near the edges (i.e., the guard band) are not modulated to achieve a sufficient transition band at the bandwidth boundaries [33].
} 
where the summation runs over the set of modulated subcarriers $n \in I_{d} \subset I_{N}, u \in\{1,2, \ldots, m\}, X_{n} \in \Omega$ and $y \in$ $\left\{1,2, \ldots, F_{u, \Re\left\{X_{n}\right\}}\right\}$ with

$$
F_{u, \Re\left\{X_{n}\right\}}=\left\lfloor\left(\frac{\Re\left\{X_{n}\right\}}{d}+M\right) 2^{-(m-u+2)}+2^{-1}\right\rfloor .
$$

In (26), $\lfloor z\rfloor$ denotes the largest integer smaller than $z$, and $d$ is the half minimum Euclidean distance between the points in $\Omega$ [32]. Further, the pre-factor $\lambda_{u, \Re\left\{X_{n}\right\}, y}$ in (25) equals

$$
\lambda_{u, \Re\left\{X_{n}\right\}, y}=(-1)^{\left\lfloor 2^{u-2-m} \cdot\left(\left(\Re\left\{X_{n}\right\}-\Delta_{u, y}\right) / d-1\right)\right\rfloor},
$$

where $\Delta_{u, y}$ are the positions of the decision boundaries, given by [19]

$$
\Delta_{u, y}=\left((2 y-1) \cdot 2^{m-u+1}-M\right) d \triangleq B_{u, y} d .
$$

Finally, the argument $\Upsilon_{u, X_{n}, y}\left(\left|\hat{H}_{n}\right|\right)$ of the complementary error function (erfc-function) is given by

$$
\begin{aligned}
& \Upsilon_{u, X_{n}, y}\left(\left|\hat{H}_{n}\right|\right) \\
& \quad=\left(\left(1+D G_{0}\right) \Re\left\{X_{n}\right\}-\Delta_{u, y}\right) \sqrt{\frac{E_{s}}{2 \sigma_{\Lambda}^{2}\left(X_{n}, \hat{H}_{n}\right)}},
\end{aligned}
$$

where $\sigma_{\Lambda}^{2}\left(X_{n}, \hat{H}_{n}\right)$ is defined in (24). As the BER of the $I$ and $Q$ components of an $M^{2}$-QAM constellation is the same, the obtained BER expression will also hold for an $M$-PAM constellation.

To obtain the overall BER, the $B E R_{\mid \hat{\mathrm{H}}}$ from (25) needs to be averaged over the statistics of $\left\{\left|\hat{H}_{n}\right|\right\}$, i.e.,

$$
B E R=\int_{0}^{+\infty} B E R_{\mid \hat{\mathbf{H}}} p_{\left|\hat{H}_{n}\right|}\left(\left|\hat{H}_{n}\right|\right) d\left|\hat{H}_{n}\right|,
$$

where $p_{\left|\hat{H}_{n}\right|}\left(\left|\hat{H}_{n}\right|\right)$ is the pdf of $\left|\hat{H}_{n}\right|$. Depending on the type of channel, we have:

1) Rayleigh channels: Using (6) and [34], the integration in (30) can be simplified to

$$
\begin{aligned}
& B E R=\frac{1}{N_{d} m M^{2}} \sum_{n, u, X_{n}, y} \lambda_{u, \Re\left\{X_{n}\right\}, y} \\
& \quad \times\left(1-\left(\left(1+D G_{0}\right) \Re\left\{X_{n}\right\}-\Delta_{u, y}\right) \sqrt{\frac{\sigma_{\hat{H}}^{2} E_{s}}{\mathcal{D}_{u, X_{n}, y}}}\right),
\end{aligned}
$$

where $\mathcal{D}_{u, X_{n}, y}$ is given by:

$$
\begin{aligned}
\mathcal{D}_{u, X_{n}, y} & =\hat{\sigma}_{\Lambda_{1, n}}^{2}+\hat{\sigma}_{\Lambda_{2, n}}^{2}+\hat{\sigma}_{\Lambda_{3, n}}^{2} \\
& +E_{s} \sigma_{\hat{H}}^{2}\left(\left(1+D G_{0}\right) \Re\left\{X_{n}\right\}-\Delta_{u, y}\right)^{2},
\end{aligned}
$$

with $\hat{\sigma}_{\Lambda_{1, n}}^{2}=\left|\hat{H}_{n}\right|^{2} \sigma_{\Lambda_{1, n}}^{2}, \hat{\sigma}_{\Lambda_{2, n}}^{2}=\left|\hat{H}_{n}\right|^{2} \sigma_{\Lambda_{2, n}}^{2}$ and $\hat{\sigma}_{\Lambda_{3, n}}^{2}=\left|\hat{H}_{n}\right|^{2} \sigma_{\Lambda_{3, n}}^{2}$. The expression (31) provides an efficient and fast approach to evaluate the BER performance compared to a brute-force Monte-Carlo (MC) computation, which can be very time consuming. A quick count learns that evaluating (31) for $\mathscr{K}$ signalto-noise ratio (SNR) values requires $\mathcal{O}\left(M^{2}\right)+\mathcal{O}(L)+$ $\mathcal{O}(\mathscr{K})$ elementary operations, whereas a MC method would require $\sum_{i=1}^{\mathscr{K}} \Psi_{i} \cdot(\mathcal{O}(M)+\mathcal{O}(L))$ operations.
TABLE II

Simulation PARAMETERS

\begin{tabular}{|c|c|}
\hline Parameters & Reference values \\
\hline$E_{s}$ & 1 \\
\hline$N$ & $64,128,512,2048$ \\
\hline$L$ & $2,3,4,6,7,8$ \\
\hline$d g_{l}^{100 \%}$ & {$[0.61,-0.75,-0.31,0.26,0.82,-0.55,-0.16,-0.95]$} \\
\hline \multirow{2}{*}{$D G_{i}^{100 \%}$} & {$[-0.1288,-0.1509-0.0705 j, 0.2375+0.0763 j$} \\
& $0.0984-0.1080 j, 0.3688,0.0984+0.1080 j$, \\
& $0.2375-0.0762 j,-0.1509+0.0705 j]$ \\
\hline
\end{tabular}

Here, $\Psi_{i}$ denotes the number of system simulations required for the MC simulation to obtain a good approximation of the BER at the $i$-th SNR value. For example, to evaluate the BER of an optical communication system of $10^{-9}$ (or lower) [2], we need to generate at least $10^{10}$ transmitted bits in case of brute-force MC method. Hence, for a given SNR, the number of the required operations for MC equals $\frac{10^{10}}{2 N \log _{2} M}(\mathcal{O}(M)+\mathcal{O}(L))$. This number is much larger than the number of operations required for evaluating (31), which equals $\mathcal{O}\left(M^{2}\right)+$ $\mathcal{O}(L)$ for a given SNR.

2) Wired channels: Using (7), the BER can be obtained by the numerical integration of (30).

\section{NUMERICAL VALIDATION AND DISCUSSION}

In this section, we validate the accuracy of the derived BER expression for Rayleigh fading and wired channels by comparing the numerical evaluation of our analytical expressions with brute-force $\mathrm{MC}$ simulation results for various constellations, and different $L, \sigma_{U}^{2}$ and mismatch levels. We assume all subcarriers are modulated, i.e., $N_{d}=N$. Further, we generate 8 independent gain errors $d g_{l}^{100 \%}$ according to a uniform distribution over the interval $[-1,1][15]$ and keep these gain errors fixed. These $L$ values can be interpreted as $100 \%$ of the gain mismatch level of a particular TI-ADC realization. Moreover, when $L<8$, only the first $L$ values of the 8 fixed gain errors will be employed. The simulation parameters are summarized in Table II. The level of mismatch will be varied by scaling the $d g_{l}^{100 \%}$ gain errors, i.e., for an $x \%$ mismatch level, we use as the gain errors: $d g_{l}^{x \%}=\frac{x}{100} d g_{l}^{100 \%}$, and $D G_{i}^{x \%}=\frac{x}{100} D G_{i}^{100 \%}$. The obtained BER is plot against the SNR per bit, i.e., $\frac{E_{b}}{N_{0}}$. The relationship between the SNR per symbol $\left(\frac{E_{s}}{N_{0}}\right)$ and the $\operatorname{SNR}$ per bit $\left(\frac{E_{b}}{N_{0}}\right)$ is given by: $\frac{E_{s}}{N_{0}}=\frac{2 m E_{b}}{N_{0}}$ for QAM and $\frac{E_{s}}{N_{0}}=\frac{m E_{b}}{N_{0}}$ for PAM. The channel impulse response is modelled as

$$
h_{k}=C e^{-\frac{1}{2 \xi}} \mathcal{A}_{k}, \quad k=0,1, \ldots, \xi-1,
$$

where $\xi$ denotes the number of channel taps. Depending on the type of channel, we distinguish two cases:

1) A Rayleigh channel: $\mathcal{A}_{k}$ are independently complexvalued random variables with standard normal distribution, i.e., $\mathcal{A}_{k} \sim \aleph(0,1)$, and $C$ is the normalization constant so that $\sum_{k=0}^{\xi-1} E\left\{\left|h_{k}\right|^{2}\right\}=1$. In this case, it is easily verified that the frequency channel coefficients $H_{n}$ are independently complex-valued random variables with standard normal distribution, i.e., $H_{n} \sim \aleph(0,1)$, 
and $H_{n}$ is also statistically independent from $\hat{H}_{n^{\prime}}$ for $n \neq n^{\prime}$.

2) A wired channel: the deterministic complex-valued coefficients $\mathcal{A}_{k} \sim \aleph(0,1)$ are generated once and kept fixed over the simulations, and $C$ is the normalization constant so that $\sum_{k=0}^{\xi-1}\left|h_{k}\right|^{2}=1$.

The obtained BER curves reflect the error performance of a given TI-ADC realization and a given channel estimator.

\section{A. Rayleigh channel}

We first consider a Rayleigh fading channel. In Fig. 2, the BER performance of a system impaired by gain mismatch and CEE is shown for different constellations, $L$ and mismatch levels. For comparison, the BER of a system without gain mismatch and CEE is also provided. It can be observed from Fig. 2 that when either the modulation order or the mismatch level increases, the BER performance significantly deteriorates, i.e., the induced error floors strongly increase. Most importantly, Fig. 2 shows that the analytical BER curves are in excellent agreement with the simulated BER curves. We also investigated numerous other parameter settings (results not shown in this paper), and found the same excellent agreement between analytical expression and simulations. Such a good agreement was to be expected considering that in the case of Rayleigh fading no approximation is involved in the derivation of the analytical BER expression (31).

Next, we compare our approach with the approach from [26]. Assuming a Rayleigh fading channel, Fig. 3 depicts the BER curves for 4-QAM, 16-QAM and 64-QAM when $N=2048, L=8$ and for $0 \%$ gain mismatch. CEE variances $\sigma_{U}^{2}$ equal to 0 (no CEE), $10^{-4}, 10^{-3}, 10^{-2}, 10^{-1}$ and 1 are considered. For large $\sigma_{U}^{2}$, the BER expression proposed in [26] does not match the simulations. The observed deviation is a result of the fact that the derivation in [26] considers the dominating BER terms only. In contrast, as is evident from Fig. 3 , the theoretical BER derived in this paper exactly predicts the simulated BER for any value of the CEE variance.

\section{B. Wired channel}

We now evaluate the derived BER expressions for wired channels. In Fig. 4, the BER curves are provided for different constellations, values of $L$, mismatch levels and CEE variances $\sigma_{U}^{2}$. For the sake of comparison, the BER without gain mismatch and CEE is also shown. We make the following observations:

- Integer $\frac{N}{L}$, no CEE: Fig. 4(a) shows that the analytical BER curves do not match the simulated BER curves when $L$ is small, i.e., $L=2,4$. However, when $L$ increases, the deviation between theory and simulation decreases. When $L=8$, the analytical result is in good agreement with the simulation. The deviation between analytical result and simulation can be explained as follows. With integer ratios $\frac{N}{L}$, we have that $\Lambda_{1, n}=$ $\frac{\sqrt{E_{s}}}{\hat{H}_{n}} \sum_{i \in I_{L} \backslash 0} D G_{i} X_{p_{i, n}} H_{p_{i, n}}$ (see (17)), i.e., $\Lambda_{1, n}$ is the summation of only $L$ terms. Hence, $\Lambda_{1, n}$ can only be approximated as a Gaussian random variable for large (a)

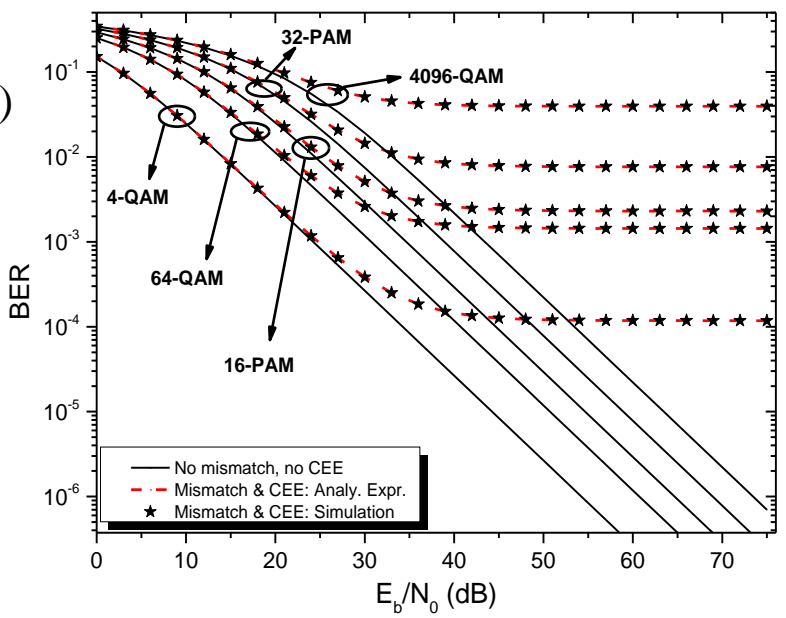

(b)

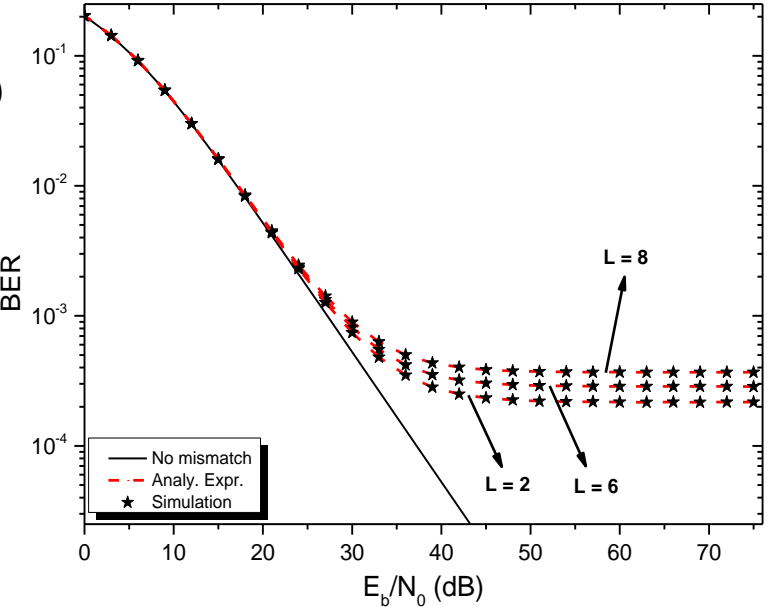

(c)

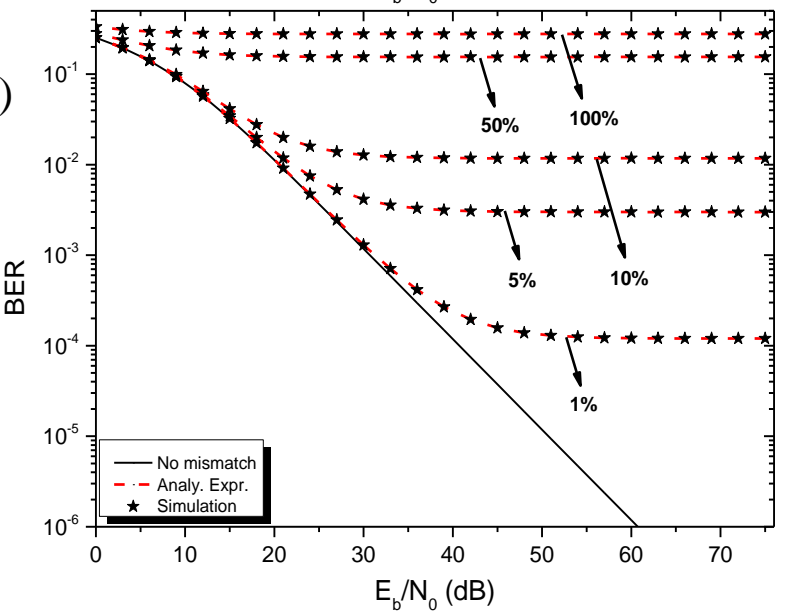

Fig. 2. BER curves for a Rayleigh channel with $N=2048$

(a) $L=8,1 \%$ mismatch and $\sigma_{U}^{2}=10^{-4}$ for square QAM and PAM

(b) 16-QAM, $2 \%$ mismatch level and no CEE when $L$ equals 2,6 and 8

(c) 8-PAM, $L=7$ and no CEE with different mismatch levels

enough values of $L$. Otherwise, the generalized central limit theorem does not apply.

- Non-integer $\frac{N}{L}$, no CEE: Fig. 4(a) shows that the analytical BER curve is in good agreement with the simulated BER curve even when $L$ is as small as 3 . This can be explained by the fact that with non-integer ratios $\frac{N}{L}$, the contribution of the gain mismatch is spread over all subcarriers. In contrast to the case of integer ratios $\frac{N}{L}$, in (17) 
(a)

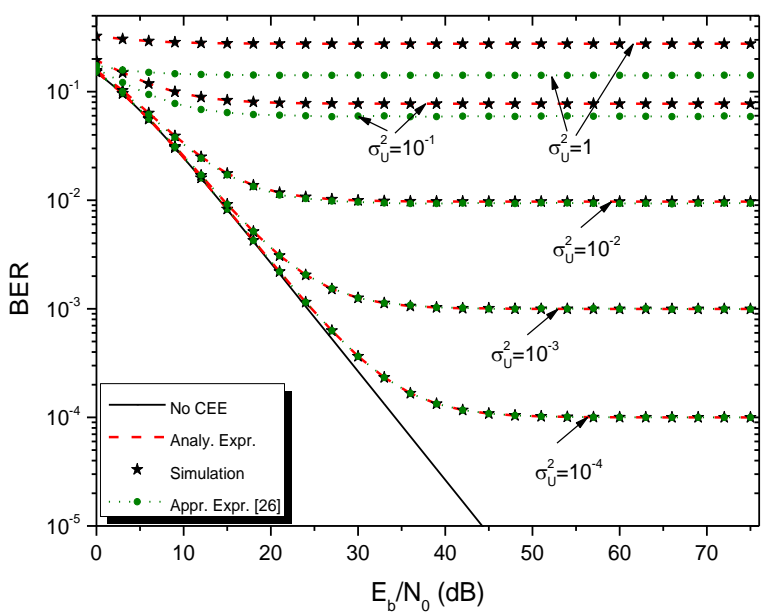

(b)

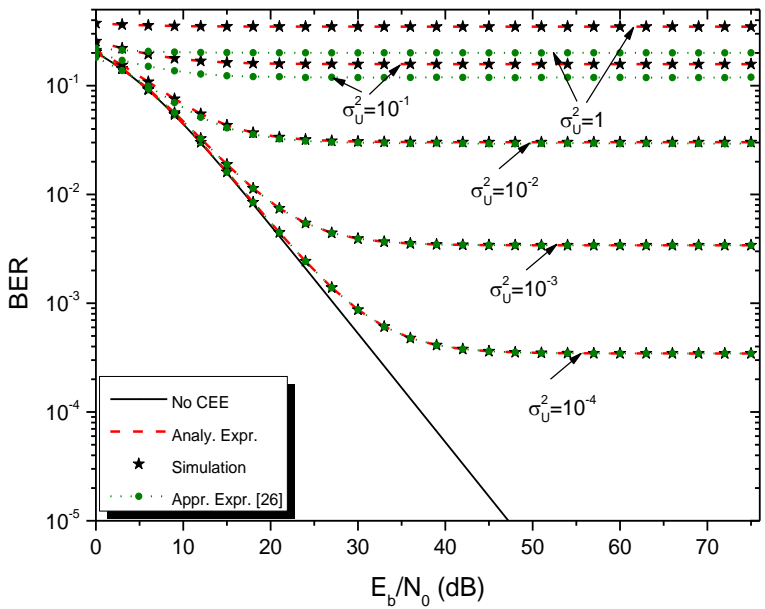

(c)

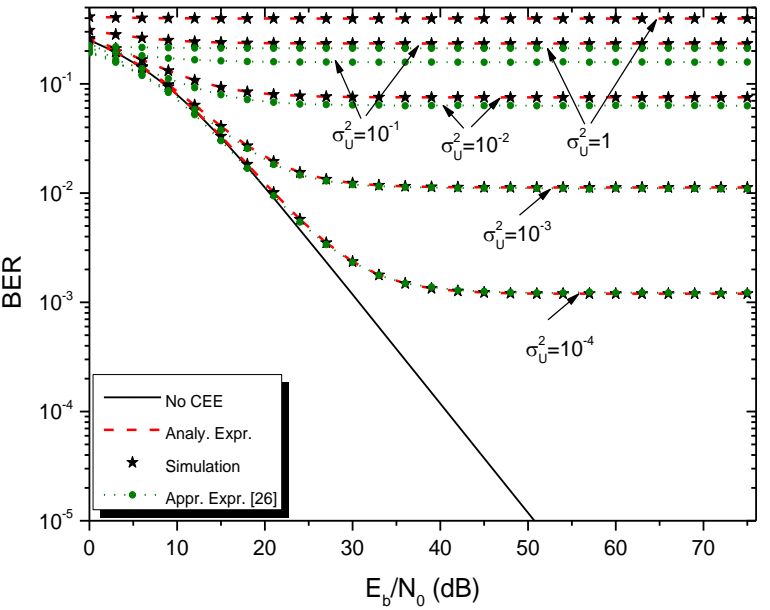

Fig. 3. BER curves for a Rayleigh fading channel for $N=2048, L=8$ and $0 \%$ gain mismatch with different values of CEE variance: (a) 4-QAM, (b) 16-QAM and (c) 64-QAM.

the summation over $N$ does not disappear. Therefore, $\Lambda_{1, n}$ consists of a summation over a large number of terms, implying that it can be approximated as a Gaussian random variable as soon as $N$ is sufficient large. Further, Fig. 4(b) indeed reveals that analytical BER curves do not match the simulations when $N=64,128$, i.e., the Gaussian approximation no longer holds. The deviation between theory and simulation reduces as $N$ increases. For $N=512$, the analytical BER curve matches well the (a)
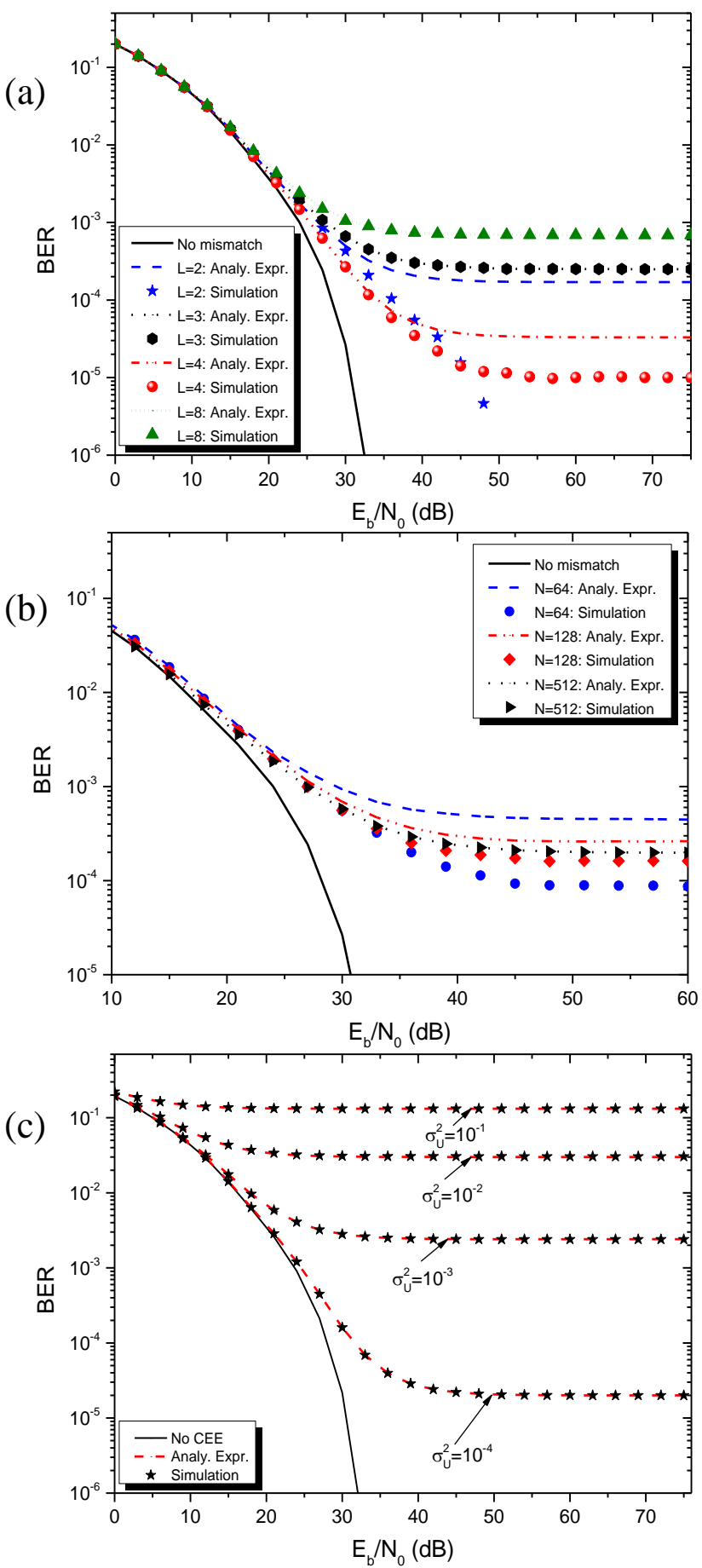

Fig. 4. BER curves for a wired channel

(a) 16-QAM, $N=2048$, no CEE and $10 \%$ mismatch with different $L$

(b) 16-QAM, $L=3$, no CEE and $10 \%$ mismatch with different $N$

(c) 16-QAM, $N=2048, L=8,0 \%$ mismatch with different values of CEE variance

simulated BER curve in Fig. 4(b).

- No gain mismatch, only CEE: Fig. 4(c) shows a good agreement between analytical expression and simulation in the absence of gain mismatch because in this case there is no approximation involved in the derivation of the BER expression: $\Lambda_{n}=-\frac{\sqrt{E_{s}}}{\hat{H}_{n}} X_{n} U_{n}+\frac{W_{n}}{\hat{H}_{n}}$ is indeed Gaussian distributed for a given $X_{n}$ and $\hat{H}_{n}$. 
Note that the results for an AWGN channel presented in [19] depicted a similar BER behaviour. This was to be expected since an AWGN channel is a special case of a wired channel, i.e., $H_{n}=1$ and $\sigma_{U}^{2}=0$.

Last but not least, it should be noticed that 1) the highspeed OFDM systems that are usually employed for broadband applications typically have thousands of active sub-carriers [35] and 2) in the coming years, the number of sub-ADCs in TI-ADC architectures will further increase to obtain extremely high sampling rates. Under these circumstances, the approximate BER expression derived in this paper is a useful tool to evaluate the BER performance in wired channels. Finally, we would like to point out that even when the obtained BER for wired channels is less accurate for integer ratios $\frac{N}{L}$ and small $L$, or for non-integer ratios $\frac{N}{L}$, small $L$ and small $N$, the derived BER expression can still serve as a useful upper bound on the true BER.

\section{Rule-OF-Thumb For a TOlerable Gain Mismatch LEVEL}

In the previous section, we focused on the accuracy of the derived BER expressions. Although this assessment of the accuracy is important, the circuit design engineer is more interested in identifying the tolerable level of the gain mismatch and the CEE that cause an acceptable level of BER performance degradation. The derivation of tolerable gain mismatch levels in the presence of a CEE is not trivial and out of the scope of this paper. The reason for this is that, in practical systems, a higher level of TI-ADC gain mismatch may result in more severe channel estimation errors, which in turn result in a higher BER. This leverage effect is difficult to model and depends on the estimator used. To simplify the analysis, we derive tolerable gain mismatch levels in the absence of a CEE, i.e., $\hat{\mathbf{H}}=\mathbf{H}$ and $\sigma_{\Lambda_{3, n}}^{2}$ (23) equals 0 . The tolerable level of the CEE has already been investigated in the literature separately [36], [37].

We define the tolerable level of gain mismatch $\tilde{\gamma}_{F} \%$ as the maximum level of gain mismatch for which the BER degradation as compared to the case without gain mismatch is smaller than $0.5 \mathrm{~dB}$ at a target BER, i.e., $B E R_{t}$. We first derive this tolerable level for a wired channel (i.e., fixed $H_{n}$ ), and later extend the results to a Rayleigh channel. In the derivation, the gain errors are assumed to be fixed and the level of mismatch is varied by scaling the $d g_{l}^{100 \%}$ gain errors.

The results shown in this paper demonstrate that a gain mismatch always introduces an error floor at high $\frac{E_{b}}{N_{0}}$. Therefore, $\tilde{\gamma}_{F} \%$ will evidently be lower than the maximum level $\gamma_{F} \%$ of the gain mismatch for which the error floor at high $\frac{E_{b}}{N_{0}}$ is smaller than $B E R_{t}$. Taking into account (25), to guarantee an error floor below $B E R_{t}$ at high SNRs (i.e., $\sigma_{\Lambda_{2, n}}^{2}$ is negligible in (24)) so that $\sigma_{\Lambda}^{2}\left(X_{n}, H_{n}\right)$ equals $\left.\sigma_{\Lambda_{1, n}}^{2}(20)\right)$, it is sufficient to demand that

$$
A \max _{n, u, v, y} \Psi\left(\tilde{\gamma}_{F} \%\right) \leq B E R_{t},
$$

where $n \in I_{N}, u \in\{1,2, \ldots, m\}, v \in\{0,1, \ldots, M-1\}, y \in$ $\left\{1,2, \ldots, F_{u, \Re\left\{X_{n}\right\}}\right\}$ with $F_{u, \Re\left\{X_{n}\right\}}(26), A=\frac{M-1}{M m}$ denotes the number of dominating BER terms at high $\frac{E_{b}}{N_{0}}$ in (25) [32], and $\Psi\left(\tilde{\gamma}_{F} \%\right)$ is given by

$$
\begin{aligned}
& \Psi\left(\tilde{\gamma}_{F} \%\right) \\
& =\operatorname{erfc}\left(\left(S_{v} d-B_{u, y} d+D G_{0}^{\tilde{\gamma}_{F} \%} S_{v} d\right)\left|H_{n}\right| \sqrt{\frac{E_{s}}{2 \hat{\sigma}_{\Lambda_{1, n}}^{2}\left(\tilde{\gamma}_{F} \%\right)}}\right)
\end{aligned}
$$

with

$$
S_{v} d \triangleq \Re\left\{X_{n}\right\}=(2 v+1-M) d,
$$

$B_{u, y}$ as defined in (28), and $\hat{\sigma}_{\Lambda_{1, n}}^{2}\left(\tilde{\gamma}_{F} \%\right)$ given by

$$
\begin{aligned}
\hat{\sigma}_{\Lambda_{1, n}}^{2}\left(\tilde{\gamma}_{F} \%\right)= & \left|H_{n}\right|^{2} \sigma_{\Lambda_{1, n}}^{2}\left(\tilde{\gamma}_{F} \%\right) \\
= & E_{s} \sum_{i_{1}, i_{2} \in I_{L} \backslash 0} D G_{i_{1}}^{\tilde{\gamma}_{F} \%}\left(D G_{i_{2}}^{\tilde{\gamma}_{F} \%}\right)^{*} \\
& \quad \times \sum_{a \in I_{N}, a \neq n} A_{a} f\left(a-p_{i_{1}, n}\right) f\left(a-p_{i_{2}, n}\right) .
\end{aligned}
$$

As the complementary error function $\operatorname{erfc}(z)$ is a monotonically decreasing function of its argument $z$, we can rewrite (34) as

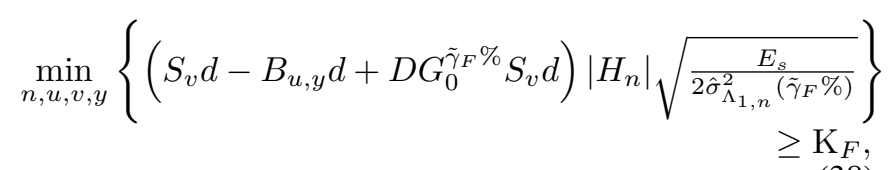

where $\mathrm{K}_{F}=\operatorname{erfc}^{-1}\left(\frac{B E R_{t}}{A}\right)$, with $\operatorname{erfc}^{-1}(z)$ the inverse complementary error function. In (38), $S_{v} d-B_{u, y} d$ corresponds to a distance between received constellation points and bit decision boundaries. The minimum value of these distances is $d$, so (38) can be rewritten as

$$
\left\{\begin{aligned}
d+D G_{0}^{\tilde{\gamma}_{F} \%} d \min _{v} S_{v} \geq \frac{\mathrm{K}_{F}}{\min _{n}\left\{\left|H_{n}\right| \sqrt{\frac{E_{s}}{2 \tilde{\sigma}_{\Lambda_{1, n}}^{2}\left(\tilde{\gamma}_{F} \%\right)}}\right\}} \\
d+D G_{0}^{\tilde{\gamma}_{F} \%} d \max _{v} S_{v} \geq \frac{, \text { if } D G_{0} \geq 0}{\min _{n}\left\{\left|H_{n}\right| \sqrt{\frac{\mathrm{K}_{F}}{2 \tilde{\sigma}_{\Lambda_{1, n}}^{2}\left(\tilde{\gamma}_{F} \%\right)}}\right\}} \\
, \text { if } D G_{0}<0
\end{aligned}\right.
$$

As $\min _{v} S_{v}=3-M$ and $\max _{v} S_{v}=M-1$, it follows that $d+\left|D G_{0}^{\tilde{\gamma}_{F} \%}\right| d \min _{v} S_{v}>d-\left|D G_{0}^{\tilde{\gamma}_{F} \%}\right| d \max _{v} S_{v}=$ $d-\left|D G_{0}^{\tilde{\gamma}_{F} \%}\right| d(M-1)$. As a result, we obtain the sufficient condition

$$
\frac{\mathrm{K}_{F}}{\left(d-\left|D G_{0}^{\tilde{\gamma}_{F} \%}\right| d(M-1)\right) \min _{n}\left\{\left|H_{n}\right| \sqrt{\frac{E_{s}}{2 \hat{\sigma}_{\Lambda_{1, n}}^{2}\left(\tilde{\gamma}_{F} \%\right)}}\right\}} \leq 1 .
$$

The inequality (40) depends on the gain mismatch level through the terms $D G_{0}^{\tilde{\gamma}_{F} \%}$ and $\hat{\sigma}_{\Lambda_{1, n}}^{2}\left(\tilde{\gamma}_{F} \%\right)$. Taking into account that $D G_{0}^{\tilde{\gamma}_{F} \%}=\frac{\tilde{\gamma}_{F}}{100} D G_{0}^{100 \%}$ and $\hat{\sigma}_{\Lambda_{1, n}}^{2}\left(\tilde{\gamma}_{F} \%\right)=$ $\left(\frac{\tilde{\gamma}_{F}}{100}\right)^{2} \hat{\sigma}_{\Lambda_{1, n}}^{2}(100 \%)$, the largest value of $\tilde{\gamma}_{F} \%$ for which (40) 


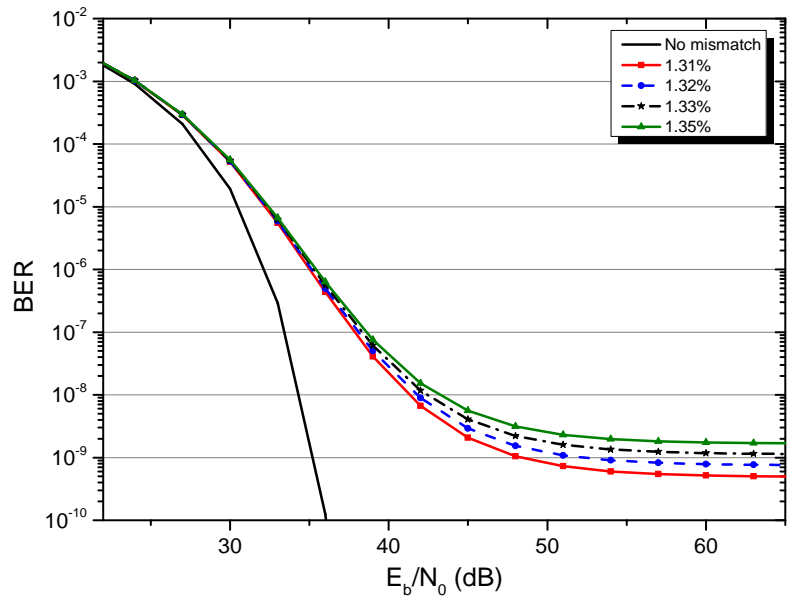

Fig. 5. Analytical BER curves in a wired channel for 16-QAM, $N=2048$, $L=8, \sigma_{U}^{2}=0$ and different mismatch levels.

holds is given by

$$
\gamma_{F} \%=\frac{100 \times d \min _{n}\left\{\left|H_{n}\right| \sqrt{\frac{E_{s}}{2 \hat{\sigma}_{1, n}^{2}(100 \%)}}\right\}}{\mathrm{K}_{F}+\left|D G_{0}^{100 \%}\right| d(M-1) \min _{n}\left\{\left|H_{n}\right| \sqrt{\frac{E_{s}}{2 \hat{\sigma}_{\Lambda_{1, n}}^{2}(100 \%)}}\right\}} \% .
$$

To demonstrate the accuracy of this threshold, we consider the BER for 16-QAM in a wired channel ${ }^{3}$, where the target $\mathrm{BER}^{4}$ equals $B E R_{t}=10^{-9}, N=2048$ and $L=8$. From (41) and Table II, we obtain $\gamma_{F} \%=1.3245 \%$. In Fig. 5, we show the BER computed with our approximation for the simulation parameters outlined in Table II and different values of $\tilde{\gamma}_{F} \%$. As can be observed, for $\tilde{\gamma}_{F} \%=1.31 \%$ and $\tilde{\gamma}_{F} \%=1.32 \%$, the error floor is below $B E R_{t}=10^{-9}$, while for $\tilde{\gamma}_{F} \%=$ $1.33 \%$ and $\tilde{\gamma}_{F} \%=1.35 \%$, the BER floor exceeds $B E R_{t}$. We also considered other constellation types, other numbers of sub-ADCs, other gain errors and other wired channels, and found the same accuracy of the threshold $\gamma_{F} \%$. Hence, we can conclude that the threshold (41) is a sufficient condition to force the error floor caused by gain mismatch below $B E R_{t}$ in a wired channel.

Starting from the threshold level $\gamma_{F} \%$ (41), we now search for the maximum gain mismatch level $\tilde{\gamma}_{F} \%$ that causes a degradation of less than $0.5 \mathrm{~dB}$ at the target BER of $B E R_{t}$. To this end, we evaluate for the simulation parameters outlined in Table II and for several values of $\tilde{\gamma}_{F} \% \leq \gamma_{F} \%$ the BER degradation compared to the case without gain mismatch. The result is shown in Fig. 6 for $N=2048, L=8$ and $\sigma_{U}^{2}=0$. For each of the constellation sizes considered in Fig. 6, the value of $\gamma_{F} \%$ is different, but the figure reveals that in all considered cases, if $\tilde{\gamma}_{F} \%$ is below $0.25 \gamma_{F} \%$, the degradation at the BER of $10^{-9}$ is at a tolerable level. This is successfully checked for other modulation types and orders, other number of sub-ADCs, and for other wired channels (without CEE).

\footnotetext{
${ }^{3}$ This wired channel in Fig. 5 is the same as the wired channel used in Fig. 4.

${ }^{4}$ This BER value is the standard target BER for optical communication systems [2].
}

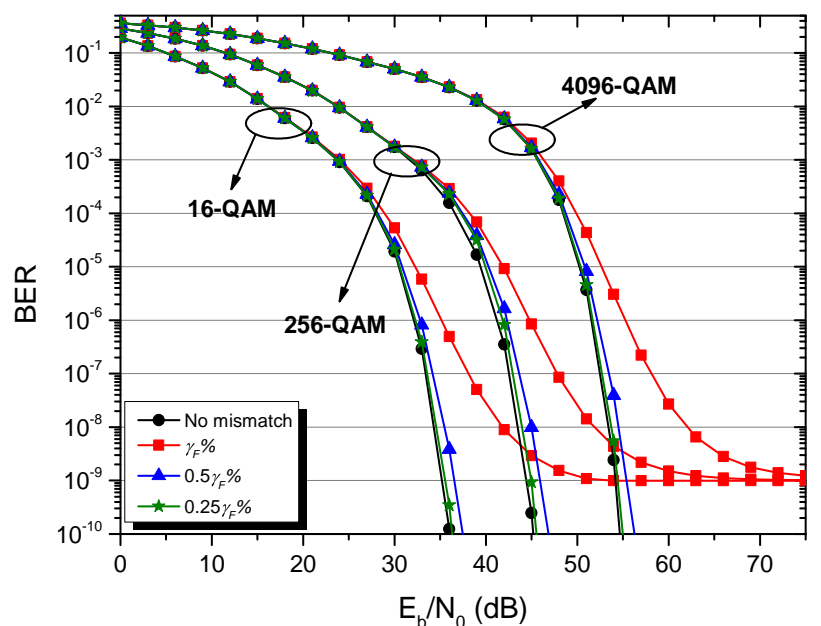

Fig. 6. Analytical BER curves in a wired channel for $N=2048, L=8$, $\sigma_{U}^{2}=0$, different modulation orders and mismatch levels.

Hence, as a rule-of-thumb, for wired channels, the tolerable level of gain mismatch is below $25 \%$ of the threshold $\gamma_{F} \%$ (41); i.e.,

$$
\tilde{\gamma}_{F} \% \leq 0.25 \gamma_{F} \% \text {. }
$$

Now we will extend the derivation of the tolerable gain mismatch level to the case of a Rayleigh channel (also without CEE, i.e., $\sigma_{U}^{2}=0$ ). Similarly as in the case of the wired channel, we will first derive the threshold $\gamma_{R} \%$ as the maximum level of gain mismatch for which the BER floor is lower than a target $B E R_{t}$, and later investigate a maximum tolerable gain mismatch level $\tilde{\gamma}_{R} \% \leq \gamma_{R} \%$ that causes a BER performance degradation of less than $0.5 \mathrm{~dB}$ at $B E R_{t}$. Taking into account (25) and (30), to obtain an error floor at high $\frac{E_{b}}{N_{0}}$ (i.e., $N_{0}=0$ ) is lower than $B E R_{t}$, it is sufficient to demand that

$$
A \max _{n, u, v, y} \int_{0}^{+\infty} \Psi\left(\tilde{\gamma}_{R} \%\right) \times \frac{\left|H_{n}\right|}{\sigma_{H}^{2}} e^{-\frac{\left|H_{n}\right|^{2}}{2 \sigma_{H}^{2}}} d\left|H_{n}\right| \leq B E R_{t},
$$

where $A=\frac{M-1}{M m}, \Psi\left(\tilde{\gamma}_{R} \%\right)$ is defined as $\Psi\left(\tilde{\gamma}_{F} \%\right)$ (35) with $D G_{0}^{\tilde{\gamma}_{F} \%}$ replaced by $D G_{0}^{\tilde{\gamma}_{R} \%}$ and $\hat{\sigma}_{\Lambda_{1, n}}^{2}\left(\tilde{\gamma}_{F} \%\right)$ replaced by $\hat{\sigma}_{\Lambda_{1, n}}^{2}\left(\tilde{\gamma}_{R} \%\right)$. In the following, we assume that $A$ is larger than $B E R_{t}$, as is typically the case. This implies that if $S_{v} d-$ $B_{u, y} d+D G_{0}^{\tilde{\gamma}_{R} \%} S_{v} d \leq 0$, the condition (43) can never be met because the left hand side of the inequality (43) becomes $\varsigma A$ with $\varsigma \geq 1$ (as $\operatorname{erfc}(z) \geq 1$ when $z \leq 0$ ), so that a tolerable gain mismatch level does not exist in this case. To satisfy (43), it is therefore required that

$$
S_{v} d-B_{u, y} d+D G_{0}^{\tilde{\gamma}_{R} \%} S_{v} d>0 .
$$

Taking into account (44) and using the integration formula in [34], (43) becomes

$$
\begin{aligned}
& \max _{n, u, v, y}\left\{1-\sqrt{\frac{\left(S_{v} d-B_{u, y} d+D G_{0}^{\tilde{\gamma}_{R} \%} S_{v} d\right)^{2} \sigma_{H}^{2} E_{s}}{\hat{\sigma}_{\Lambda_{1, n}}^{2}\left(\tilde{\gamma}_{R} \%\right)+\sigma_{H}^{2} E_{s}\left(S_{v} d-B_{u, y} d+D G_{0}^{\tilde{\gamma}_{R} \%} S_{v} d\right)^{2}}}\right\} \\
& \leq \frac{B E R_{t}}{A}
\end{aligned}
$$


or equivalently

$$
\min _{n, u, v, y}\left\{\sqrt{\frac{\left(S_{v} d-B_{u, y} d+D G_{0}^{\tilde{\gamma}_{R} \%} S_{v} d\right)^{2}}{\hat{\sigma}_{\Lambda_{1, n}}^{2}\left(\tilde{\gamma}_{R} \%\right)+\sigma_{H}^{2} E_{s}\left(S_{v} d-B_{u, y} d+D G_{0}^{\tilde{\gamma} R}{ }^{\%} S_{v} d\right)^{2}}}\right\}
$$

where $\mathrm{K}_{R}=\left(\sigma_{H}^{2} E_{s}\right)^{-1 / 2}\left(1-\frac{B E R_{t}}{A}\right)$. In (46), the only term depending on $n$ is $\hat{\sigma}_{\Lambda_{1, n}}^{2}\left(\tilde{\gamma}_{R} \%\right)$. Hence, minimizing the left hand side of the inequality (46) with respect to $n$ is equivalent to

$$
\min _{u, v, y}\left\{\sqrt{\frac{\left(S_{v} d-B_{u, y} d+D G_{0}^{\tilde{\gamma} R^{\%}} S_{v} d\right)^{2}}{\max _{n} \hat{\sigma}_{\Lambda_{1, n}}^{2}\left(\tilde{\gamma}_{R} \%\right)+\sigma_{H}^{2} E_{s}\left(S_{v} d-B_{u, y} d+D G_{0}^{\tilde{\gamma}}{ }^{\%} S_{v} d\right)^{2}}}\right\}
$$

Further, (47) can be rewritten as:

$$
\min _{u, v, y}\left\{\left(\frac{\max _{n} \hat{\sigma}_{\Lambda_{1, n}}^{2}\left(\tilde{\gamma}_{R} \%\right)}{\left(S_{v} d-B_{u, y} d+D G_{0}^{\tilde{\gamma}_{R} \%} S_{v} d\right)^{2}}+\sigma_{H}^{2} E_{s}\right)^{-\frac{1}{2}}\right\}
$$

Minimizing the left hand side of (48) with respect to $u, v, y$ is equivalent to minimizing $S_{v} d-B_{u, y} d+D G_{0}^{\tilde{\gamma}_{R} \%} S_{v} d$ over $u$, $v, y$. As discussed in the case of a wired channel, the minimum value of $S_{v} d-B_{u, y} d+D G_{0}^{\tilde{\gamma}_{R} \%} S_{v} d$ with respect to $u, v, y$ equals $d-\left|D G_{0}^{\tilde{\gamma}_{R} \%}\right| d(M-1)$. Hence, (48) reduces to

$$
\mathrm{K}_{R} \times\left(\frac{\max _{n} \hat{\sigma}_{\Lambda_{1, n}}^{2}\left(\tilde{\gamma}_{R} \%\right)}{\left(d-\left|D G_{0}^{\tilde{\gamma}_{R} \%}\right| d(M-1)\right)^{2}}+\sigma_{H}^{2} E_{s}\right)^{\frac{1}{2}} \leq 1
$$

Using (49), we now determine the threshold $\gamma_{R} \%$ for which the error floor at high $\frac{E_{b}}{N_{0}}$ induced by the gain mismatch is lower than the target $B E R_{t}$, i.e., if we scale $d g_{l}^{100 \%}$ from Table II as $\tilde{\gamma}_{R} \% \times d g_{l}^{100 \%}$ with $\tilde{\gamma}_{R} \% \leq \gamma_{R} \%$, $B E R_{E_{b} / N_{0} \rightarrow+\infty} \leq B E R_{t}$. To find the threshold $\gamma_{R} \%$, we solve the quadratic equation in $z=\gamma_{R} \%$ obtained by considering the equality in (49), i.e., $a_{1} z^{2}+a_{2} z+a_{3}=0$, with

$$
\begin{aligned}
& a_{1}=\max _{n} \hat{\sigma}_{\Lambda_{1, n}}^{2}(100 \%) \mathrm{K}_{R}^{2} \\
& \quad-\left|D G_{0}^{100 \%}\right|^{2} d^{2}(M-1)^{2}\left(1-\sigma_{H}^{2} E_{s} \mathrm{~K}_{R}^{2}\right), \\
& a_{2}=2\left|D G_{0}^{100 \%}\right| d^{2}(M-1)\left(1-\sigma_{H}^{2} E_{s} \mathrm{~K}_{R}^{2}\right)
\end{aligned}
$$

and

$$
a_{3}=\sigma_{H}^{2} E_{s} d^{2} \mathrm{~K}_{R}^{2}-d^{2} .
$$

The threshold $\gamma_{R} \%$ is the positive-valued root that is closest to 0 . Taking into account that $a_{1} \neq 0, a_{2}>0, a_{3}<0$ and $a_{2}^{2}-4 a_{1} a_{3}>0$, it can be easily verified that this threshold equals

$$
\gamma_{R} \%=\frac{100 \times\left(-a_{2}+\sqrt{a_{2}^{2}-4 a_{1} a_{3}}\right)}{2 a_{1}} \% .
$$

Similarly to the case of a wired channel, it can be verified numerically that the maximum tolerable gain mismatch level

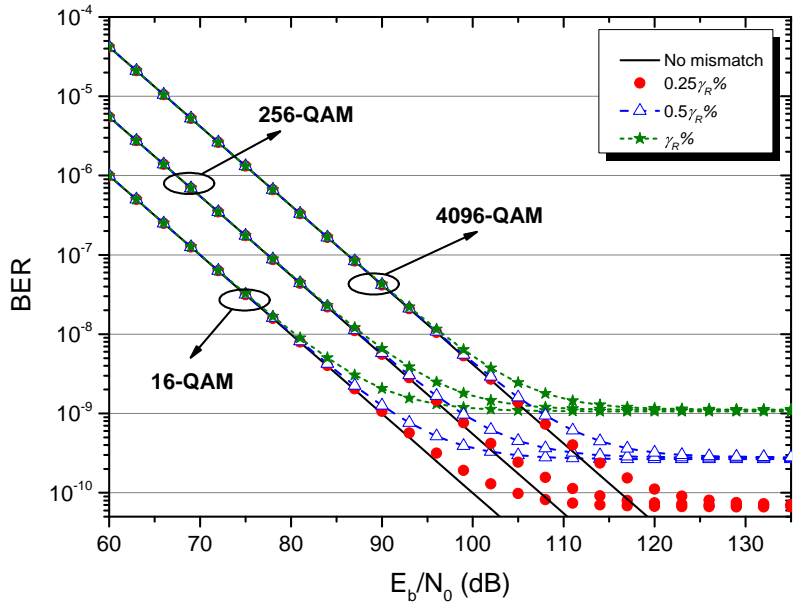

Fig. 7. Analytical BER curves in a Rayleigh channel for $N=2048, L=8$, $\sigma_{U}^{2}=0$, different modulation type and mismatch levels.

$\tilde{\gamma}_{R} \%$ causing an acceptable BER performance degradation in a Rayleigh channel equals $0.25 \gamma_{R} \%$; i.e.,

$$
\tilde{\gamma}_{R} \% \leq 0.25 \gamma_{R} \% \text {. }
$$

For example, Fig. 7 shows the analytical BER in a Rayleigh channel for the simulation parameters from Table II and for the different modulation types and mismatch levels used in Fig. 2 when $B E R_{t}=10^{-9}, N=2048, L=8$ and $\sigma_{U}^{2}=0$. As can be observed from the figure, when the mismatch level equals $0.25 \gamma_{R} \%$, the degradation at $B E R_{t}=10^{-9}$ is imperceptible, whereas a degradation is visible when the mismatch level increases to $\gamma_{R} \%$. This has also been checked successfully for other modulation orders and types and other number of sub-ADCs. Hence, (54) is the proposed rule-of-thumb for a tolerable level of the gain mismatch in Rayleigh channels.

\section{RESUlTS FOR RANDOM GAIN ERRORS}

Up to now, the obtained results are based on the fixed gain errors $d g_{l}$ from Table II. However, in reality, the gain errors are random variables. Hence, we now evaluate the average performance in the case of random gain errors, which can be done by averaging the obtained BER performance over different TI-ADC realizations. Fig. 8 illustrates the averaged BER performance in a Rayleigh fading channel and a wired channel $^{5}$ without CEE when the gain errors are randomly selected from $[-x / 100, x / 100]$ with $0 \leq x \leq 100$, which corresponds to $x \%$ mismatch level (i.e., we generate different sets of $L$ gain errors, where each set corresponds to a different TI-ADC realization). The results in Fig. 8 are plotted for 16QAM, $10^{5}$ TI-ADC realizations, $B E R_{t}=10^{-9}, \sigma_{U}^{2}=0$, $N=2048, L=8$ and different gain mismatch levels. First, as expected, Fig. 8(a) shows a good agreement between analytical expression and simulation for the case of a Rayleigh channel, and Fig. 8(b) reveals a deviation between analytical expression and simulation for the case of a wired channel with $\gamma_{F} \%$ mismatch level at high $\frac{E_{b}}{N_{0}}$. Next, most importantly, as can be

\footnotetext{
${ }^{5}$ This wired channel in Fig. 8 is the same as the wired channel used in Fig.
} 4. 

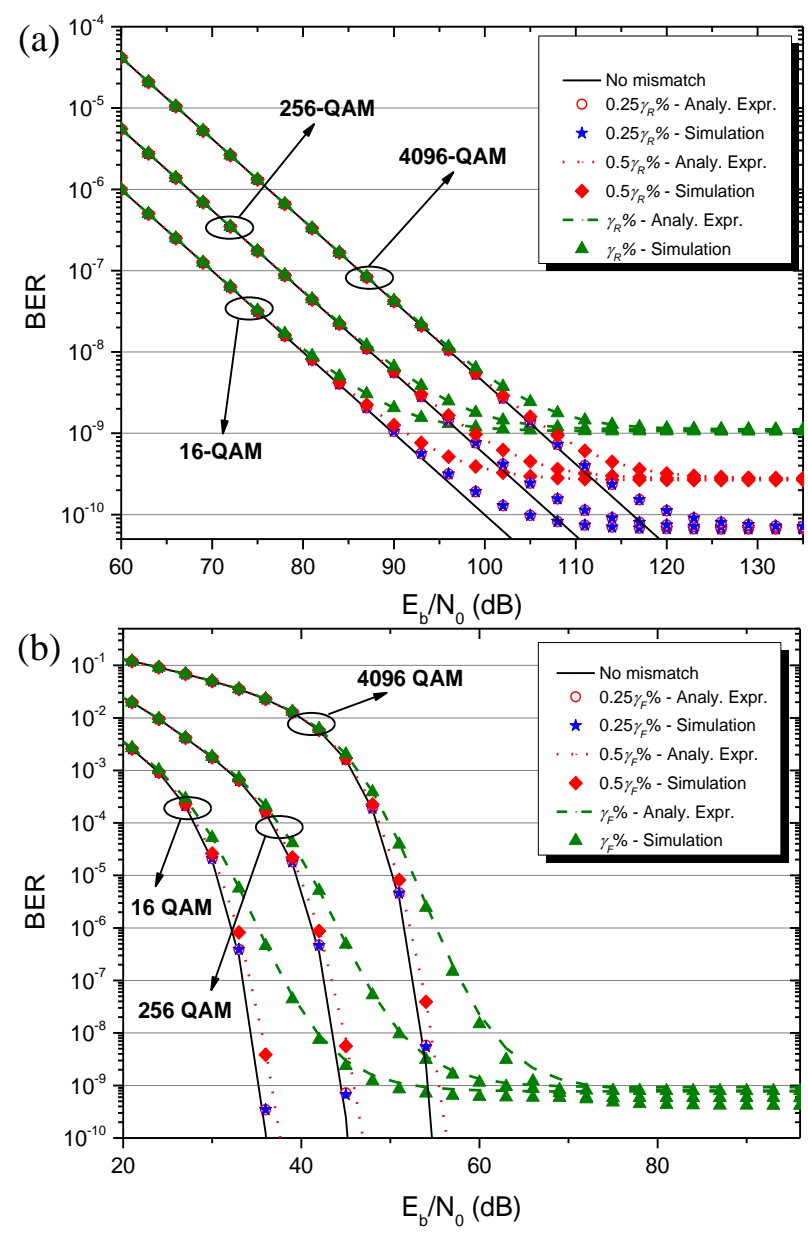

Fig. 8. BER curves for 16-QAM with $B E R_{t}=10^{-9}, N=2048, L=8$, $\sigma_{U}^{2}=0$, different mismatch level and $5 \times 10^{3}$ sets of $L$ random gain errors: (a) a Rayleigh channel, (b) a wired channel.

seen from Fig. 8, when the gain mismatch level equals the tolerable degradation threshold $0.25 \gamma_{F} \%$ for a wired channel and $0.25 \gamma_{R} \%$ for a Rayleigh channel, where $\gamma_{F} \%$ and $\gamma_{R} \%$ are defined in (41) and (53), respectively, there is no visible degradation with respect to the 'no mismatch' case at $B E R_{t}$, whereas a degradation is noticed when the mismatch level is increased to $\gamma_{F} \%$ for a wired channel or $\gamma_{R} \%$ for a Rayleigh channel at $B E R_{t}=10^{-9}$. Therefore, the proposed rule-ofthumb can be used in any wired or Rayleigh fading channels.

\section{CONCLUSIONS}

In this paper, we proposed an analytical approach to evaluate the BER of PAM- and square QAM-OFDM systems impaired by the joint effect of TI-ADC gain mismatch and CEEs in Rayleigh fading channels and wired channels, based on modelling the inter-carrier interference caused by gain mismatch and CEE as Gaussian distributed. Further, based on the obtained BER expressions, we were able to analytically determine the gain mismatch level at which the error floor caused by the gain mismatch is below a target BER value $B E R_{t}$ at high SNRs. We showed in this paper that, if and only if we select the gain mismatch level to be less than $25 \%$ of the proposed threshold level, the BER performance degradation at $B E R_{t}$ is less than $0.5 \mathrm{~dB}$ with respect to the mismatch-free case. Based on our findings, engineers designing TI-ADCs for high-speed OFDM applications are able to extract the maximum gain mismatch level that can be tolerated. This can serve as an important guideline for calibration and compensation of this type of mismatch.

\section{ACKNOWLEDGMENT}

This research has been funded by the EOS programme of the Flemish Research Council (FWO) through the grant 30452698.

\section{REFERENCES}

[1] R. Prasad, OFDM for Wireless Communications Systems. Artech House, 2004.

[2] J. Armstrong, "OFDM for optical communications," Journal of Lightwave Technology, vol. 27, no. 3, pp. 189-204, Feb 2009.

[3] "Wireless PAN high rate alternative PHY task group IEEE 802.15 3a," http://www.ieee802.org/15/pub/TG3a.html.

[4] "Wireless PAN high rate alternative PHY task group IEEE 802.15 3c," http://www.ieee802.org/15/pub/TG3c.html.

[5] W. C. Black and D. A. Hodges, "Time interleaved converter arrays," IEEE Journal of Solid-State Circuits, vol. 15, no. 6, pp. 1022-1029, Dec 1980.

[6] R. Khoini-Poorfard, L. B. Lim, and D. A. Johns, "Time-interleaved oversampling A/D converters: theory and practice," IEEE Transactions on Circuits and Systems II: Analog and Digital Signal Processing, vol. 44, no. 8, pp. 634-645, Aug 1997.

[7] D. Fu, K. C. Dyer, S. H. Lewis, and P. J. Hurst, "A digital background calibration technique for time-interleaved analog-to-digital converters,' IEEE Journal of Solid-State Circuits, vol. 33, no. 12, pp. 1904-1911, Dec 1998.

[8] A. Petraglia and S. K. Mitra, "Analysis of mismatch effects among A/D converters in a time-interleaved waveform digitizer," IEEE Transactions on Instrumentation and Measurement, vol. 40, no. 5, pp. 831-835, Oct 1991.

[9] C. Vogel, "The impact of combined channel mismatch effects in timeinterleaved ADCs," IEEE Transactions on Instrumentation and Measurement, vol. 54, no. 1, pp. 415-427, Feb 2005.

[10] C. A. Schmidt, J. E. Cousseau, J. L. Figueroa, B. T. Reyes, and M. R. Hueda, "Efficient estimation and correction of mismatch errors in time-interleaved ADCs," IEEE Transactions on Instrumentation and Measurement, vol. 65, no. 2, pp. 243-254, Feb 2016.

[11] S. Xu, Y. C. Lim, and J. W. Lee, "Recursive filters for time-interleaved ADC mismatch compensation," IEEE Transactions on Circuits and Systems I: Regular Papers, vol. 63, no. 6, pp. 848-858, June 2016.

[12] S. Chen, L. Wang, H. Zhang, R. Murugesu, D. Dunwell, and A. C. Carusone, "All-digital calibration of timing mismatch error in timeinterleaved analog-to-digital converters," IEEE Transactions on Very Large Scale Integration (VLSI) Systems, vol. 25, no. 9, pp. 2552-2560, Sept 2017.

[13] P. Monsurr, F. Rosato, and A. Trifiletti, "New models for the calibration of four-channel time-interleaved ADCs using filter banks," IEEE Transactions on Circuits and Systems II: Express Briefs, vol. 65, no. 2, pp. 141-145, Feb 2018.

[14] Y. Oh and B. Murmann, "System embedded ADC calibration for OFDM receivers," IEEE Transactions on Circuits and Systems I: Regular Papers, vol. 53, no. 8, pp. 1693-1703, Aug 2006.

[15] S. Ponnuru, M. Seo, U. Madhow, and M. Rodwell, "Joint mismatch and channel compensation for high-speed OFDM receivers with timeinterleaved ADCs," IEEE Transactions on Communications, vol. 58, no. 8, pp. 2391-2401, August 2010.

[16] K. M. Tsui and S. C. Chan, "Iterative correction of frequency response mismatches in time-interleaved ADCs: A novel framework and case study in OFDM systems," in The 2010 International Conference on Green Circuits and Systems, June 2010, pp. 253-258.

[17] V. T. D. Huynh, N. Noels, P. Rombouts, J. Armstrong, and H. Steendam, "Effect of time-interleaved analog-to-digital converter mismatches on OFDM performance," in OFDM 2014; 18th International OFDM Workshop 2014 (InOWo'14); Proceedings of, Aug 2014, pp. 1-8.

[18] V. T. D. Huynh, N. Noels, and H. Steendam, "Effect of offset mismatch in time-interleaved ADC circuits on OFDM-BER performance," IEEE Transactions on Circuits and Systems I: Regular Papers, vol. 64, no. 8, pp. 2195-2206, Aug 2017. 
[19] V. T. D. Huynh, N. Noels, and H. Steendam, "Approximate BER for OFDM systems impaired by a gain mismatch of a TI-ADC realization," in 2017 24th International Conference on Telecommunications (ICT), May 2017, pp. 1-6.

[20] V. T. D. Huynh, N. Noels, and H. Steendam, "Accurate and efficient BER evaluation for high-speed OFDM systems impaired by TI-ADC circuit's gain mismatch," in 2017 23rd Asia-Pacific Conference on Communications (APCC), Dec 2017, pp. 1-6.

[21] X. Tang, M. S. Alouini, and A. Goldsmith, "Effect of channel estimation error on M-QAM BER performance in Rayleigh fading," in 1999 IEEE 49th Vehicular Technology Conference, vol. 2, Jul 1999, pp. 1111-1115 vol.2.

[22] L. Cao and N. C. Beaulieu, "Exact error-rate analysis of diversity 16QAM with channel estimation error," IEEE Transactions on Communications, vol. 52, no. 6, pp. 1019-1029, June 2004

[23] K. Yu, J. Evans, and I. Collings, "Performance analysis of pilot symbol aided QAM for Rayleigh fading channels," in 2002 IEEE International Conference on Communications. Conference Proceedings. ICC 2002, vol. 3, 2002, pp. 1731-1735 vol.3.

[24] M. X. Chang and Y. T. Su, "Performance analysis of equalized OFDM systems in Rayleigh fading," IEEE Transactions on Wireless Communications, vol. 1, no. 4, pp. 721-732, Oct 2002.

[25] M. Al-Gharabally and P. Das, "On the performance of OFDM systems in time varying channels with channel estimation error," in 2006 IEEE International Conference on Communications, vol. 11, June 2006, pp. $5180-5185$.

[26] H. Cheon and D. Hong, "Effect of channel estimation error in OFDMbased WLAN," IEEE Communications Letters, vol. 6, no. 5, pp. 190 192, May 2002.

[27] C. A. Schmidt, J. E. Cousseau, J. L. Figueroa, B. T. Reyes, and M. R. Hueda, "Efficient estimation and correction of mismatch errors in time-interleaved ADCs," IEEE Transactions on Instrumentation and Measurement, vol. 65, no. 2, pp. 243-254, Feb 2016.

[28] I. Ku, Z. Xu, Y. Kuan, Y. Wang, and M. F. Chang, "A 40-mw 7-bit 2.2-gs/s time-interleaved subranging CMOS ADC for low-power gigabit wireless communications," IEEE Journal of Solid-State Circuits, vol. 47, no. 8, pp. 1854-1865, Aug 2012

[29] M. Gardner, Knotted Doughnuts and Other Mathematical Entertainments. W.H. Freeman, 1986.

[30] H. Venghaus and N. Grote, Fibre Optic Communication: Key Devices, ser. Springer series in optical sciences. Springer Berlin Heidelberg, 2012.

[31] P. D. Jong, "A central limit theorem for generalized multilinear forms," Journal of Multivariate Analysis, vol. 34, no. 2, pp. 275 - 289, 1990.

[32] K. Cho and D. Yoon, "On the general BER expression of one- and two-dimensional amplitude modulations," IEEE Transactions on Communications, vol. 50, no. 7, pp. 1074-1080, July 2002.

[33] I. Guvenc, S. Gezici, Z. Sahinoglu, and U. Kozat, Reliable Communications for Short-Range Wireless Systems. Cambridge University Press, 2011.

[34] A. Jeffrey and D. Zwillinger, Table of Integrals, Series, and Products, ser. Table of Integrals, Series, and Products Series. Elsevier Science, 2007.

[35] N. Richard and P. Ramjee, OFDM for Wireless Multimedia Communications, 1st ed. Norwood, MA, USA: Artech House, Inc., 2000.

[36] J. J. van de Beek, O. Edfors, M. Sandell, S. K. Wilson, and P. O. Borjesson, "On channel estimation in OFDM systems," in 1995 IEEE 45th Vehicular Technology Conference. Countdown to the Wireless Twenty-First Century, vol. 2, Jul 1995, pp. 815-819 vol.2.

[37] B. Jiao, J. Xiao, and J. Wang, "Analysis of channel estimation error of OFDM systems in Rayleigh fading," in International Symposium on Signals, Circuits and Systems, 2005. ISSCS 2005., vol. 2, July 2005, pp. 653-656 Vol. 2.

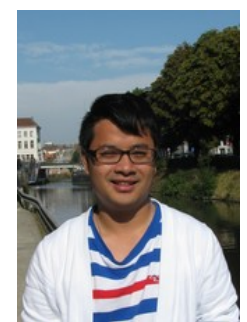

Vo-Trung-Dung Huynh was born in Vietnam, in 1987. He obtained the bachelors degree in telecommunications at Ho Chi Minh City University of Technology, Vietnam in 2010. He received the masters degree in information and mechatronics from Gwangju Institute of Science and Technology, South Korea in 2012. Since 2013, he has pursued the Ph.D. degree with the Digital Communications Research Group, Department of Telecommunications and Information Processing, Ghent University, Belgium. His main research interests are in digital communications, OFDM, BER performance analysis, and time-interleaved analogto-digital converter circuits.

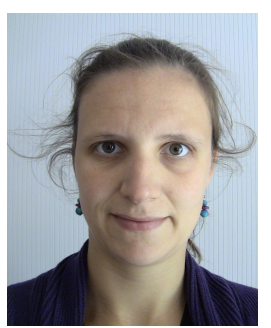

Nele Noels (S04M11) received the Diploma and the Ph.D. degrees in electrical engineering from Ghent University, Gent, Belgium, in 2001 and 2009, respectively. She is currently a Professor with the Department of Telecommunications and Information Processing, Ghent University. She has co-authored over 50 academic papers in international journals and conference proceedings. Her main research interests are in statistical communication theory, carrier and symbol synchronization, bandwidth-efficient modulation and coding, massive MIMO, optical OFDM, and satellite and mobile communication. She was the recipient of several scientific awards. In 2010, she received the Scientific Award Alcatel Lucent Bell for the best Belgian thesis concerning an original study of information and communication technology, concepts, and/or applications.

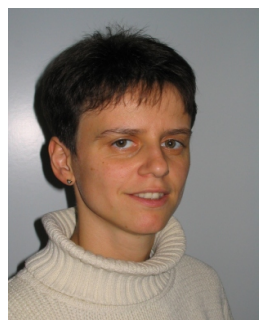

Heidi Steendam (M01, SM06) received the M.Sc. degree in Electrical Engineering and the Ph.D. degree in Applied Sciences from Ghent University, Gent, Belgium in 1995 and 2000, respectively. Since September 1995, she has been with the Digital Communications (DIGCOM) Research Group, Department of Telecommunications and Information Processing (TELIN), Faculty of Engineering, Ghent University, Belgium, first in the framework of various research projects, and since October 2002 as a Professor in the area of Digital Communications. In 2015, she was a visiting professor at Monash University. Her main research interests are in statistical communication theory, carrier and symbol synchronization, bandwidth-efficient modulation and coding, cognitive radio and cooperative networks, positioning and visible light communication. She is the author of more than 150 scientific papers in international journals and conference proceedings, for which several best paper awards were received.

Since 2002, she has been an executive Committee Member of the IEEE Communications and Vehicular Technology Society Joint Chapter, Benelux Section, since 2012 the vice chair and since 2017 the chair. She was active in various international conferences as Technical Program Committee chair/member and Session chair. In 2004 and 2011, she was the conference chair of the IEEE Symposium on Communications and Vehicular Technology in the Benelux. From 2012 till 2017, she was associate editor of IEEE Transactions on Communications and EURASIP Journal on Wireless Communications and Networking. 